\title{
Experimental study of pedestrian flow through a T-junction
}

\author{
J Zhang ${ }^{1}$, W Klingsch ${ }^{1}$, A Schadschneider ${ }^{2}$, and A Seyfried ${ }^{3,4}$ \\ 1 Institute for Building Material Technology and Fire Safety Science, Wuppertal \\ University, Pauluskirchstrasse 11, 42285 Wuppertal, Germany \\ jun.zhang@uni-wuppertal.de, klingsch@uni-wuppertal.de \\ 2 Institut für Theoretische Physik, Universität zu Köln, 50937 Köln, Germany \\ as@thp.uni-koeln.de \\ 3 Computer Simulation for Fire Safety and Pedestrian Traffic, Wuppertal \\ University, Pauluskirchstrasse 11, 42285 Wuppertal, Germany \\ 4 Jülich Supercomputing Centre, Forschungszentrum Jülich GmbH, 52425 Jülich, \\ Germany seyfried@uni-wuppertal.de
}

Summary. In this study, series of experiments under laboratory conditions were carried out to investigate pedestrian flow through a T-junction, i.e., two branches merging into the main stream. The whole duration of the experiments was recorded by video cameras and the trajectories of each pedestrian were extracted using the software Petrack from these videos. The Voronoi method is used to resolve the fine structure of the fundamental diagram and spatial dependence of the measured quantities from trajectories. In our study, only the data in the stationary state are used by analyzing the time series of density and velocity. The density, velocity and specific flow profiles are obtained by refining the size of the measurement area (here $10 \mathrm{~cm} \times 10 \mathrm{~cm}$ are adopted). With such a high resolution, the spatial distribution of density, velocity and specific flow can be obtained separately and the regions with higher value can be observed intuitively. Finally, the fundamental diagrams of T-junction flow is compared in three different locations. It is shown that the fundamental diagrams of the two branches match well. However, the velocities in front of the merging are significantly lower than that in the main stream at the same densities. After the merging, the specific flow increases with the density $\rho$ till $2.5 \mathrm{~m}^{-2}$. While in the branches, the specific flow is almost independent of the density between $\rho=1.5 \mathrm{~m}^{-2}$ and $3.5 \mathrm{~m}^{-2}$.

\section{Introduction}

In recent years, research on pedestrian and traffic flow became popular and attracted a lot of attention [1, 2, 3, 4, 5]. A great deal of models has been proposed to simulate pedestrian movement in different situations $[6,7]$. Most of these models are able to reproduce crowd phenomena such as lane formation 
qualitatively. On the other hand, some field studies and laboratory experiments are also carried out to obtain empirical data and improve models.

Unfortunately, the empirical database is insufficient leading to discrepancies among different handbooks. The fundamental diagram describing the empirical relation between density $(\rho)$, velocity $(v)$ and flow $(J)$ (or specific flow $\left.\left(J_{s}\right)\right)$ is the key characteristics of pedestrian dynamics used to support the planning and design of facilities. However, there is considerable disagreement among the empirical data from different studies and handbooks even for the same type of facility or movement. The maximum flow as well as the density where the maximum flow appear has a broad range $[8,9]$. These discrepancies cause big inconvenience in design and assessment. Further research is necessary to study the reasons for these differences. Carrying out laboratory experiments is a good way to do this, since secondary factors can be removed or controlled.

T-junctions are important part of most of buildings. In this kind of structure, bottleneck flow, merging flow or split flow are all possible to take place in different situations. Especially around the corners, pedestrian behavior is much more complex. Pedestrian flow characteristics in this kind of geometry are significant to study. However, except for some studies using a Cellular Automaton model $[10,11]$, there are few empirical studies directly considering the fundamental diagram of pedestrian flow in T-junctions.

In this study, series of well-controlled laboratory experiments are carried out to investigate the pedestrian movement in T-junctions. The Voronoi method is accepted to analyze the fundamental diagram and field profiles of density, velocity and specific flow.

\section{Experiment setup and trajectory extraction}

Seven runs of experiments were carried out in a T-junction with corridor width $b_{\text {cor } 1}=b_{\text {cor2 }}=2.4 \mathrm{~m}$. Fig. 1 shows the sketch of the experiment setup. Pedestrian streams move from two branches oppositely and then merge into the main stream at the T-junction. To regulate the pedestrian density in the corridor, the width of the entrance $b_{\text {entrance }}$ was changed in each run from $0.5 \mathrm{~m}$ to $2.4 \mathrm{~m}$, see details in Table 1 . For simplicity, the left and right entrances have the same width. In this way, we guarantee the symmetry of the two branch streams. More than 300 pedestrians participated in the experiments, which makes the duration of the run long enough to obtain stationary states. The average age and body height of the tested persons was $25 \pm 5.7$ years and $1.76 \pm 0.09 \mathrm{~m}$ (range from $1.49 \mathrm{~m}$ to $2.01 \mathrm{~m}$ ), respectively. They mostly consisted of German students of both genders. The free velocity $v_{0}=1.55 \pm 0.18 \mathrm{~m} / \mathrm{s}$ was obtained by measuring 42 participants' free movement.

At the beginning, the participants were held within waiting areas in the left and right side. When the experiment starts, they pass through a $4 \mathrm{~m}$ 
Table 1. Parameters for the T-junction experiments

\begin{tabular}{|c|c|c|c|c|c|}
\hline Runs & Name & $b_{\text {cor } 1}[m]$ & $b_{\text {entrance }}[m]$ & $b_{\text {cor2 } 2}[m]$ & $N_{l}+N_{r}$ \\
\hline 1 & $T-240-050-240$ & 2.40 & 0.50 & 2.40 & $67+67$ \\
\hline 2 & $T-240-060-240$ & 2.40 & 0.60 & 2.40 & $66+66$ \\
\hline 3 & $T-240-080-240$ & 2.40 & 0.80 & 2.40 & $114+114$ \\
\hline 4 & $T-240-100-240$ & 2.40 & 1.00 & 2.40 & $104+104$ \\
\hline 5 & $T-240-120-240$ & 2.40 & 1.20 & 2.40 & $152+153$ \\
\hline 6 & $T-240-150-240$ & 2.40 & 1.50 & 2.40 & $153+152$ \\
\hline 7 & $T-240-240-240$ & 2.40 & 2.40 & 2.40 & $151+152$ \\
\hline
\end{tabular}

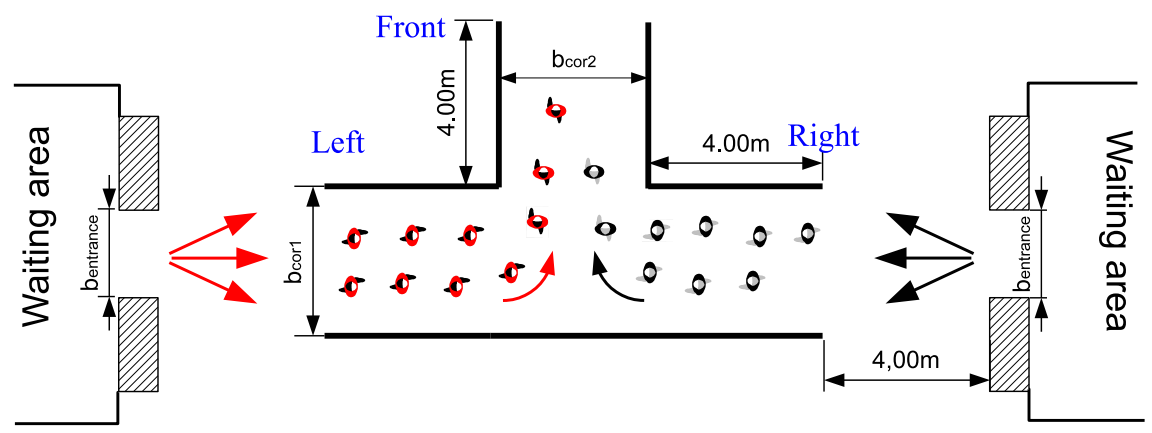

Fig. 1. Setup of T-junction experiments.

passage into the corridor simultaneously and merge into the main stream at the vertical corner. The passage was used as a buffer to minimize the effect of the entrance. In this way, the pedestrian flow in the corridor was nearly homogeneous over its entire width. When a pedestrian leaves the main corridor, he or she returned to the waiting area for the next run.

The whole processes of the experiments were recorded by two synchronized stereo cameras of type Bumblebee XB3 (manufactured by Point Grey). They were mounted on the rack of the ceiling $7.84 \mathrm{~m}$ above the floor with the viewing direction perpendicular to the floor. The cameras have a resolution of $1280 \times 960$ pixels and a frame rate of $16 \mathrm{fps}$ (corresponding to 0.0625 second per frame. To cover the complete region, the left and the right part of the scenario were recorded by the two cameras separately (see Fig. 2(a)). The overlapping field of view of the stereo system is $\alpha=64^{\circ}$ at the average head distance of about $6 \mathrm{~m}$ from the cameras. With the above-mentioned height range, all pedestrians can be seen without occlusion at any time.

To make a highly precise analysis, accurate pedestrian trajectories were automatically extracted from video recordings using the software PeTrack [12]. Lens distortion and perspective view are taken into account in this program. Fig. 2(b) shows the pedestrian trajectories for one run of the experiments. 


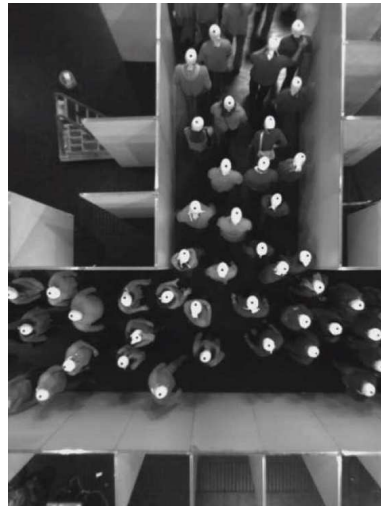

(a) Snapshot

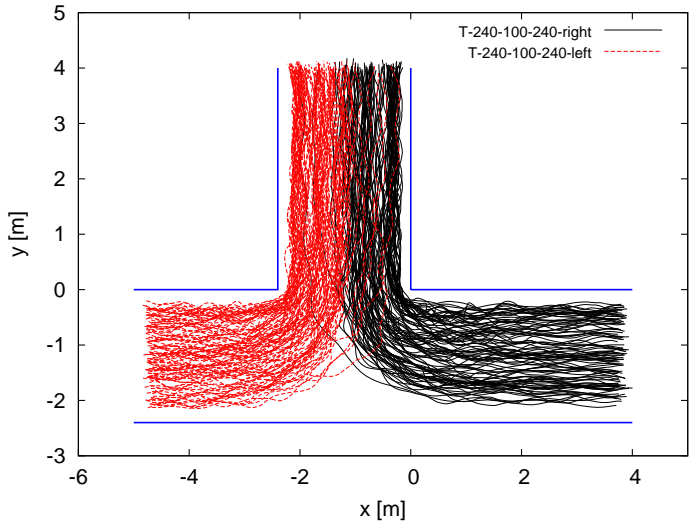

(b) Pedestrian trajectories

Fig. 2. Trajectories and snapshot from T-junction experiment.

From these trajectories, pedestrian characteristics including flow, density, velocity and individual distances at any time and position can be determined.

\section{Experiment analysis}

In previous studies, different measurement methods were used to limit the comparability and fluctuation of the data. E.G. Helbing et al. proposed a Gaussian, distance-dependent weight function [13] to measure the local density and local velocity. Predtechenskii and Milinskii [14] used a dimensionless definition to consider different body sizes and Fruin introduced the "Pedestrian Area Module" [15]. This study focuses on the Voronoi method proposed in $[16,17]$, where the density distribution can be assigned to each pedestrian. This method permits examination on scales smaller than the pedestrians for its high spatial resolution.

\subsection{Measurement methodology}

At a given time $t$, the Voronoi diagram can be generated from the positions of each pedestrian. It contains a set of Voronoi cells for each pedestrian $i$. The cell area, $A_{i}$, can be thought as the personal space belonging to each pedestrian $i$. Then, the density and velocity distribution over space can be defined as

$$
\rho_{x y}=1 / A_{i} \quad \text { and } \quad v_{x y}=v_{i}(t) \quad \text { if }(x, y) \in A_{i}
$$

where $v_{i}(t)$ is the instantaneous velocity of each person (see [17]). The Voronoi density and velocity for the measurement area $A_{m}$ is defined as 


$$
\begin{aligned}
\langle\rho\rangle_{v}(x, y, t) & =\frac{\iint \rho_{x y} d x d y}{A_{m}}, \\
\langle v\rangle_{v}(x, y, t) & =\frac{\iint v_{x y} d x d y}{A_{m}} .
\end{aligned}
$$

The specific flow

$$
J_{s}(x, y, t)=\langle\rho\rangle_{v}(x, y, t) \cdot\langle v\rangle_{v}(x, y, t)
$$

can also be calculated using the Voronoi density and velocity.

\subsection{Results and analysis}

To analyze the spatial dependence of density, velocity and specific flow precisely, we use the Voronoi method to measure these quantities in areas smaller than the size of pedestrians. We calculate the Voronoi density, velocity and specific flow over small regions $(10 \mathrm{~cm} \times 10 \mathrm{~cm})$ each frame. Then the spatiotemporal profiles of density $(\bar{\rho}(x, y))$, velocity $(\bar{v}(x, y))$ and specific flow $\left(\overline{J_{s}}(x, y)\right)$ can be obtained over the stationary state separately for each run as follows:

$$
\begin{aligned}
& \bar{\rho}(x, y)=\frac{\int_{t_{1}}^{t_{2}}\langle\rho\rangle_{v}(x, y, t) d t}{t_{2}-t_{1}}, \\
& \bar{v}(x, y)=\frac{\int_{t_{1}}^{t_{2}}\langle v\rangle_{v}(x, y, t) d t}{t_{2}-t_{1}} \\
& \overline{J_{s}}(x, y)=\bar{\rho}(x, y) \cdot \bar{v}(x, y) .
\end{aligned}
$$

Fig. 3 shows the profiles for two runs of the experiments under the situations of low density ( $T$-240-050-240) and high density (T-240-240-240), respectively. These profiles provide new insights into the spatiotemporal dynamics of the motion and the sensitivity of the quantities to other potential factors. The density distribution in T-junction is not homogeneous both for low and high density situations. For the former, the higher density region locates at the main stream after merging. Whereas for the latter, the higher density region appears near the junction and the lowest density region locates at a small triangle area, where the left and right branches begin to merge. For both of the two situations, the densities in the branches are not uniform and are higher over the inner side, especially near the corners. In other words, pedestrians prefer to move along the shorter and smoother path. Moreover the density profile shows obvious boundary effects at high density situation. The spatiotemporal variation of the velocity is different. At low density situation, the velocity profile is nearly homogeneous all over the T-junction except the places near corner. Pedestrians move in free velocity and slow down round 

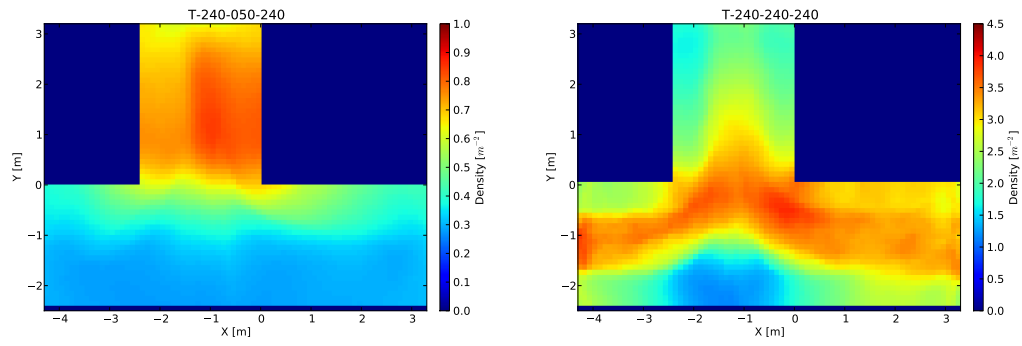

(a) Density profile
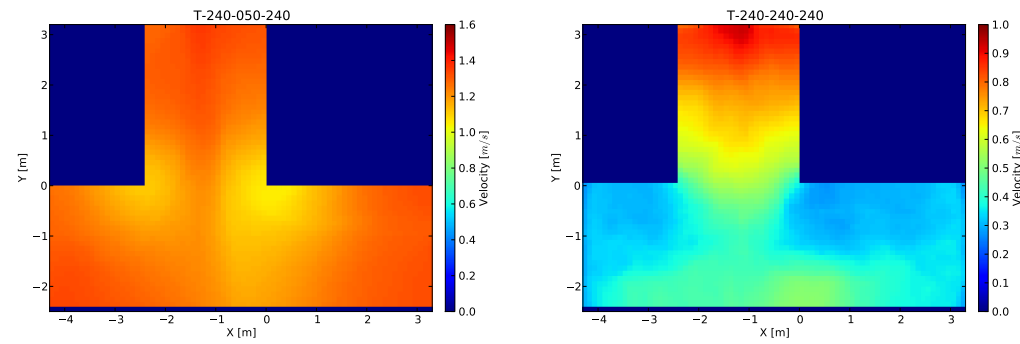

(b) Velocity profile
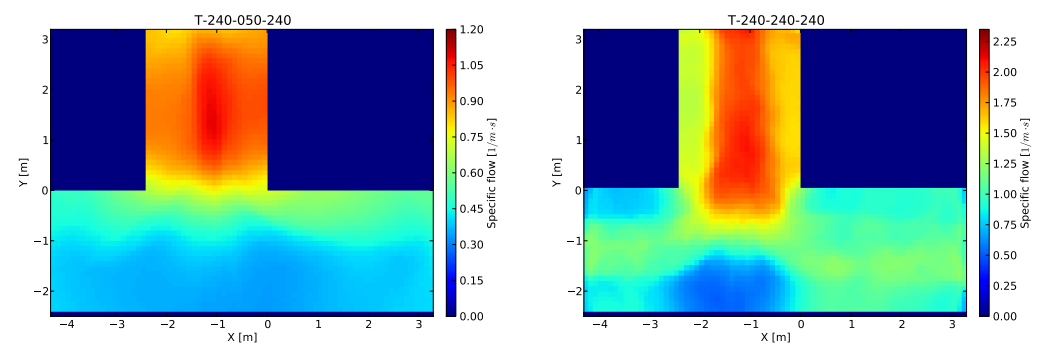

(c) Specific flow profile

Fig. 3. The profiles of density, velocity and specific flow in T-junction obtained from Voronoi method. The resolution of the profiles is $10 \mathrm{~cm} \times 10 \mathrm{~cm}$.

the corner. While for high density condition, the velocity distribution is not uniform any more. The velocities of main stream is higher obviously than that in the branches. Boundary effect does not occur and the velocities after merging increase along the movement direction persistently. By comparison of the specific flow profiles at the two different situations, the highest flow regions are both observed at the center of the main stream after the merging. The different is that the region extends further into the area where the two branches start to merge for the high density case, but for the other case it doesn't. This 
indicates that the merging process in front of the exit corridor leads to a flow restriction. Causes for the restriction of the flow must be located outside the region of highest flow.

These profiles demonstrate that density and velocity measurements are sensitive to the size and location of the measurement area. For the comparison of measurements (e.g. for model validation or calibration), it is necessary to specify precisely the size and position of the measurement area.
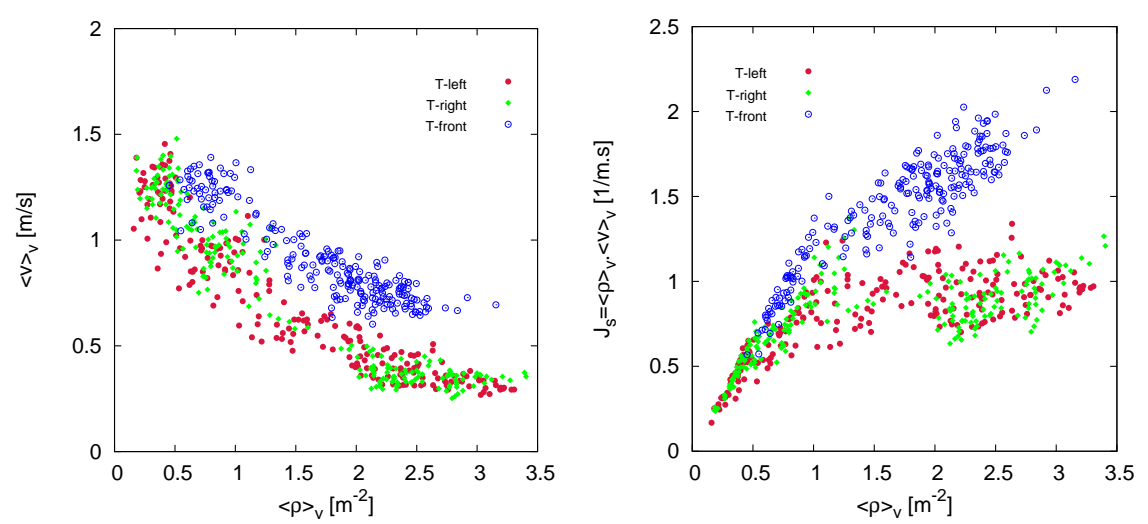

Fig. 4. Fundamental diagram of pedestrian flow at different measurement areas in T-junction.

In Fig. 4, we compare the fundamental diagrams obtained from all Tjunction experiments. The data assigned with 'T-left' and 'T-right' are measured in the areas before the streams merge, while the data assigned with ' $\mathrm{T}$ front' are measured in the region where the streams have already merged. The locations of these measurement areas can be seen in Table. 2. For ease of comparison, we choose these measurement areas with the same size $\left(4.8 \mathrm{~m}^{-2}\right)$. One finds that the fundamental diagrams of the two branches match well. However, for densities $\rho>0.5 \mathrm{~m}^{-2}$ the velocities in the 'right' and 'left' part of the T-junction (T-left and T-right) are significantly lower than the velocities measured after the merging of the streams (T-front). This discrepancy becomes more distinct in the relation between density and specific flow. In the main stream (T-front), the specific flow increases with the density $\rho$ till $2.5 \mathrm{~m}^{-2}$. While in the branches, the specific flow nearly remains constant for density $\rho$ between $1.5 \mathrm{~m}^{-2}$ and $3.5 \mathrm{~m}^{-2}$. Thus, there seems no unique fundamental diagram which describes the relation between velocity and density for the complete system.

For this difference, we can only offer assumptions regarding the causes. One is based on behavior of pedestrians. Congestion occurs at the end of the branches, where the region of maximum density appears. Pedestrians stand 
Table 2. The location of measurement area in T-junction

\begin{tabular}{cc}
\hline Measurement area & Range $[\mathrm{m}]$ \\
\hline T-left & $x \in[-4.5,-2.5], y \in[-2.4,0]$ \\
T-right & $x \in[1.0,3.0], y \in[-2.4,0]$ \\
T-front & $x \in[-2.4,0], y \in[1.0,3.0]$ \\
\hline
\end{tabular}

in a jam in front of the merging and could not perceive where the congestion disperse or whether the jam lasts after the merging. In such situation, it is questionable whether an urge or a push will lead to a benefit. Thus an optimal usage of the available space becomes unimportant. Otherwise, the situation totally changes if the location of dissolution becomes apparent. Then a certain urge or an optimal usage of the available space makes sense and could lead to a benefit. They will move in a relatively active way. That's maybe the reason why the velocities after merging are higher than that in front of merging at the same density. Whether this explanation is plausible could be answered by a comparison of these data with experimental data at a corner without the merging. This comparison is in preparation.

\section{References}

1. C. Appert-Rolland, F. Chevoir, P. Gondret, S. Lassarre, J.-P. Lebacque, and M. Schreckenberg (2009) Traffic and Granular Flow '07 (Berlin: Springer)

2. S. Bandini, S. Manzoni, H. Umeo, and G. Vizzari (2010) Workshop on Crowds and CA, ACRI 2010: Cellular Automata 9th International Conference on Cellular Automata for Reseach and Industry (Berlin: Springer), Italy

3. W. W. F. Klingsch, C. Rogsch, A. Schadschneider, and M. Schreckenberg (2010) Pedestrian and Evacuation Dynamics 2008. Springer-Verlag Berlin Heidelberg

4. A. Schadschneider, H. Klüpfel, T. Kretz, and A. Rogsch, C.and Seyfried (2009) Fundamentals of Pedestrian and Evacuation Dynamics. In: Multi-Agent Systems for Traffic and Transportation Engineering, pp. 124-154. IGI Global, Hershey, Pennsylvania, USA

5. A. Schadschneider and A. Seyfried (2009) Pedestrian Behavior: Data Collection and Applications. In. Empirical Results for Pedestrian Dynamics and their Implications for Cellular Automata Models. pp. 27-43. Emerald Group Publishing Limited, 1st edition

6. D. Helbing, I. Farkas, and T. Vicsek (2000) Nature 407:487-490

7. W. Song, X. Xu, B.H. Wang, and S. Ni (2006) Physica A 363:492-500

8. A. Schadschneider, W. Klingsch, H. Klüpfel, T. Kretz, C. Rogsch, and A. Seyfried (2009) Evacuation Dynamics: Empirical Results, Modeling and Applications. In: Encyclopedia of Complexity and System Science, volume 5, (Berlin: Springer), pp. 3142-3176

9. A. Seyfried, O. Passon, B. Steffen, M. Boltes, T. Rupprecht, and W. Klingsch (2009) Transportation Science 43(3):395-406

10. Y. Tajima and T. Nagatani (2002) Physica A 303:239-250 
11. Y.C. Peng and C.I. Chou (2011) Computer Physics Communications 182:205208

12. M. Boltes, A. Seyfried, B. Steffen, and A. Schadschneider (2010) Automatic Extraction of Pedestrian Trajectories from Video Recordings. In Pedestrian and Evacuation Dynamics 2008 (Berlin: Springer), pp. 43-54

13. D. Helbing, A. Johansson, and H. Z. Al-Abideen (2007) Phys. Rev. E 75:046109

14. V. M. Predtechenskii and A. I. Milinskii (1969) Planning for Foot Traffic Flow in Buildings. Amerind Publishing, New Dehli, 1978. Translation of: Proekttirovanie Zhdanii s Uchetom Organizatsii Dvizheniya Lyuddskikh Potokov, Stroiizdat Publishers, Moscow

15. J. J. Fruin (1971) Pedestrian Planning and Design. Elevator World, New York

16. B. Steffen and A. Seyfried (2010) Physica A 389(9):1902-1910

17. J. Zhang, W. Klingsch, A. Schadschneider, and A. Seyfried (2011) J. Stat. Mech. P06004. 